\title{
Neuroprotective Effects of Oleocanthal, a Compound in Virgin Olive Oil, in a Rat Model of Traumatic Brain Injury
}

\author{
Mesut METE ${ }^{1}$, Isil AYDEMIR ${ }^{2}$, Ulkun Unlu UNSAL ${ }^{3}$, Fatih COLLU ${ }^{4}$, Gokhan VATANDAS ${ }^{1}$, Beyhan GURCU ${ }^{4}$, \\ Yusuf Kurtulus DURANSOY ${ }^{1}$, Fatma TANELI ${ }^{5}$, Mehmet Ibrahim TUGLU6 ${ }^{6}$, Mehmet SELCUKI ${ }^{1}$ \\ ${ }^{1}$ Celal Bayar University, School of Medicine, Department of Neurosurgery, Manisa, Turkey \\ ${ }^{2}$ Omer Halisdemir University, School of Medicine, Department of Histology and Embryology, Nigde, Turkey \\ ${ }^{3}$ Koc University, School of Medicine, Department of Neurosurgery, Istanbul, Turkey \\ ${ }^{4}$ Celal Bayar University, Faculty of Science and Letters, Department of Biology, Zoology Section, Manisa, Turkey \\ ${ }^{5}$ Celal Bayar University, School of Medicine, Department of Biochemistry, Manisa, Turkey \\ ${ }^{6}$ Celal Bayar University, School of Medicine, Department of Histology and Embryology, Manisa, Turkey
}

\section{ABSTRACT}

AIM: To evaluate the neuroprotective effects of deocanthal $\mathrm{OC}$ in a rat model of traumatic brain injury (TBI).

MATERIAL and METHODS: Twenty-six adult male, Wistar albino rats were used. The rats were divided into 4 groups. Group 1 was the sham group $(n=5)$. Group 2 was the trauma group $(n=5)$ where rats were treated with $10 \mathrm{mg} / \mathrm{kg}$ saline intraperitoneally (IP) twice a day. Groups 3 and 4, rats were treated with 10 (group 3, n=8) or 30 (group 4, n=8) mg/kg OC IP twice a day. For each group, brain samples were collected 72 hours after injury. Brain samples and blood were evaluated with histopathological and biochemical methods.

RESULTS: Histopathological evaluation revealed a significant difference between Group 2 and Group 4. Biochemical findings demonstrated that the oxidative stress index was highest in Group 2 and lowest in Group 4.

CONCLUSION: OC has a protective effect on neural cells after TBI. This effect is achieved by reducing oxidative stress and apoptosis.

KEYWORDS: Apoptosis, Neuroprotection, Oleocanthal, Rat, Traumatic brain injury

\section{INTRODUCTION}

$\mathrm{T}$ Iraumatic brain injury (TBI) is a leading cause of death and lifelong disability worldwide $(4,6)$. TBI has two distinct phases: acute, primary injury and delayed, secondary injury. Primary injury occurs at the trauma instant and comprises brain damage and diffuse axonal injury. Secondary injury occurs hours to days or months after the initial trauma and includes mitochondrial dysfunction, lipid peroxidation, ionic imbalance, excessive release of neurotransmitters, and membrane degradation, all of which contribute to neuronal cell loss (20). The brain's inflammatory response to trauma is multifactorial and consists of the activation of central nervous system immune cells, cerebral infiltration of peripheral immune cells, and upregulation of inflammatory cytokines, chemokines, and reactive oxygen species (14).

Oleocanthal (OC), a phenolic component in virgin olive oil (VOO), has anti-inflammatory and antioxidant properties similar to ibuprofen, which is a non-steroidal anti-inflammatory drug (NSAID) $(1,3,19)$. Neuroprotective effects of OC against neurodegenerative conditions, such as Alzheimer's disease, have been reported (1). However, to the best of our knowledge, no study has considered the neuroprotective effects of OC 
against TBI. We evaluated the neuroprotective effects of OC in a rat model of TBI using histopathological and biochemical methods.

\section{MATERIAL and METHODS}

\section{Experimental Protocol}

In the present study, 26 adult, male, Wistar albino rats $(200 \pm$ $50 \mathrm{~g})$ were used. The rats were kept under a temperature of $22^{\circ} \mathrm{C}$, humidity of $65 \%$, and light-dark cycles of $12: 12 \mathrm{~h}$, and they had ad libitum access to laboratory standard food and water. This study was approved by The Animal Experiments Local Ethics Committee of Manisa Celal Bayar University (06/12/2016/77.637.435-75), Turkey.

The rats were grouped as follows:

Group 1, sham $(n=5)$, skin incision only: a skin incision was made, and non-traumatic brain samples were collected 72 hours after surgery.

Group 2, trauma $(n=5)$ : TBI was induced as described below, and rats were treated with $10 \mathrm{mg} / \mathrm{kg}$ saline intraperitoneally (IP) twice a day. After craniectomy, brain samples were collected 72 hours after injury.

Groups 3 and 4: TBI was induced, rats were treated with 10 (Group 3, n=8) or 30 (Group 4, n=8) mg/kg OC IP twice a day, and brain samples were collected 72 hours after injury.

The rats in the treated groups (Groups 2, 3 and 4) received the first dose immediately after trauma and other doses were given at 12-hour intervals for 72 hours. For all treated groups vehicle or $\mathrm{OC}$ were given with a total of 6 doses.

\section{Anesthesia and Trauma Procedure}

Before the surgical procedure, the rats received an IP injection of $10 \mathrm{mg} / \mathrm{kg}$ xylazine (Bayer Birleşik Alman Ilaç Fabrikaları, Istanbul, Turkey) and $75 \mathrm{mg} / \mathrm{kg}$ ketamine hydrochloride (Parke Davis, Istanbul, Turkey). TBI was induced using the falling weight technique described by Marmarou et al. and modified by Ucar et al. (25). Briefly, each anesthetized rat's scalp was shaved, a midline incision was made, and the periosteum was retracted. A 2-mm thick metallic disc was used as the helmet. The steel disc was fixed to the skull's central portion using bone wax. The rats were placed in the prone position on a foam bed. An inflexible rope was tied to the weight to prevent repeated impacts. A 350-g steel weight was dropped through a 1-m vertical section of the plexiglass tube. After 3 days, the rats were sacrificed. Brain tissues were immediately extracted without damaging the tissues, and intracardiac blood samples were collected. Neural tissues were fixed in a $10 \%$ formalin solution for 24-48 hours (9), tissues were embedded in paraffin blocks. Five-micrometer-thick sections were cut using a microtome, and they were stained with histochemical, immunohistochemical, and terminal deoxynucleotidyl transferase dUTP nick end labeling (TUNEL) assays. Cardiac blood samples were collected for biochemical evaluations.

\section{Biochemical Evaluations}

Before sacrificing the animals, cardiac blood samples were centrifuged for 5 minutes at $4,500 \mathrm{r} / \mathrm{min}$ at $4^{\circ} \mathrm{C}$ to separate the serum and plasma. Serum Total Antioxidant Status (TAS) and Total Oxidant Status (TOS) levels were determined using an automated analyzer (ChroMate Manager 4300, Palm City, FL, USA). The values are expressed in terms of micromolar hydrogen peroxide equivalent per liter $\left(\mu \mathrm{mol} \mathrm{H}_{2} \mathrm{O}_{2} \mathrm{Eq} / \mathrm{L}\right)(7,8)$. The Oxidative Stress Index (OSI) is an indicator of the degree of oxidative stress. The ratio of TOS to TAS represents the OSI. The OSI is calculated according to the formula (12) in which TAS values are converted to $\mu \mathrm{mol} / \mathrm{L}$ :

$$
\begin{gathered}
\text { OSI (arbitrary unit) }=\left[\operatorname{TOS}\left(\mu \mathrm{mol} \mathrm{H}_{2} \mathrm{O}_{2} \mathrm{Eq} / \mathrm{L}\right) / \mathrm{TAS}\right. \\
(\mu \mathrm{mol} \text { Trolox Eq/L) }] \times 100
\end{gathered}
$$

Table I and Figure 5 show the OSIs of the four groups.

\section{Histochemistry}

Sections were incubated at $60^{\circ} \mathrm{C}$ overnight and deparaffinized in xylene for 30 minutes. Then, they were passed through an alcohol series, decreasing from $95 \%$ to $60 \%$ for rehydration, and washed under running water for 5 minutes. The sections were stained with hematoxylin (01562E; Surgipath, Bretton, Peter Borough, Cambridgeshire) for 2 minutes and then washed under water for 5 minutes to remove the excess dye from the tissue. The sections were stained with eosin (01602E; Surgipath, Bretton) for 30 seconds. After washing under running water for 5 minutes, air-dried sections were passed through $80 \%$ and $95 \%$ alcohol series and dewaxed in xylene for 30 minutes. The sections were mounted with entellan (UN 1866; Merck, Darmstadt, Germany). Differences among the groups were evaluated by a blinded observer using an Olympus BX40 light microscope (Figure 1).

\section{Cresyl Violet Stain}

Cresyl Violet staining is used to identify neuronal loss. Sections were incubated at $60^{\circ} \mathrm{C}$ overnight and deparaffinized in xylene for 1 hour. Then the sections were passed through decreasing alcohol series of $95 \%$ to $60 \%$ for the rehydration process and washed with distilled water. Then, the samples were stained with Cresyl Violet for 20 minutes. After washing under water for 5 minutes, sections were passed through $80 \%$ and $95 \%$

Table I: The Oxidative Stress Index (OSI) Values in Rat's Blood

\begin{tabular}{lcccc}
\hline Subjects & Sham & Trauma & OC 10 mg & OC 30 mg \\
\hline Subject 1 & 672.46 & 837.22 & 433.33 & 435.83 \\
\hline Subject 2 & 495.71 & 811.72 & 497.74 & 522.22 \\
\hline Subject 3 & 479.48 & 663.76 & 700 & 283.89 \\
\hline Subject 4 & 450.38 & 660.76 & 365.74 & 179.28 \\
\hline Subject 5 & 173.85 & 342.34 & 703.12 & 690.90 \\
\hline Subject 6 & & & 308.87 & 169.49 \\
\hline Subject 7 & & & 238.46 & 160 \\
\hline Subject 8 & & & 428.49 & 299.57 \\
\hline $\begin{array}{l}\text { Average } \\
\text { OSI }\end{array}$ & 454.38 & 663.16 & 459.47 & 342.65 \\
\hline
\end{tabular}


alcohol series quickly, and taken placed in xylene for 1 hour. The samples were mounted with entellan (UN 1866, Merck, Darmstadt, Germany). Differences between the groups were evaluated by a blinded observer under an Olympus BX40 light microscope (Figure 1A-H).

\section{Immunohistochemistry}

The sections were incubated at $60^{\circ} \mathrm{C}$ overnight and then in xylene for $\mathbf{3 0}$ minutes for immunohistochemical staining. After washing with distilled water for 5 minutes, the sections were rehydrated with an alcohol series at a rate decreasing from $95 \%$ to $60 \%$. They were then washed in phosphate buffered saline (PBS) for 5 minutes and were treated with $0.5 \%$ trypsin solution (800.729.8350, ScyTek Laboratories, Inc., Logan, UT, USA) for 15 minutes at $37^{\circ} \mathrm{C}$ and then with $3 \% \mathrm{H}_{2} \mathrm{O}_{2}$ for 5 minutes to inhibit tissue endogenous peroxidase. Following washing in a blocking solution (TA-125-UB; Lab Vision, Fremont, CA, USA) for 1 hour, the sections were incubated for 18 hours at $4^{\circ} \mathrm{C}$ with the primary antibodies antiendothelial nitric oxide synthase (anti-eNOS; sc-654; Santa Cruz Biotechnology, Dallas, TX, USA), anti-inducible nitric oxide synthase (iNOS) (GTX15322, GeneTex, Inc., Irvine, CA, USA), anti-vascular endothelial growth factor (VEGF) (ab1316; Abcam, Cambridge, United Kingdom), and anti-S100 (sc58839, Santa Cruz Biotechnology). After incubation with the primary antibodies, the sections were washed three times for 5 minutes with PBS and were then stained with anti-mouse biotin-streptavidin hydrogen peroxidase secondary antibody (85-9043 Zymed Histostain kit; Zymed, San Francisco, CA, USA) for 30 minutes. The sections were washed three times in PBS for 5 minutes and were then stained with diaminobenzidine (DAB; ScyTek) for 5 minutes to determine the appearance of the immunohistochemical reaction.
Counterstaining was performed using Mayer's hematoxylin (72804E; Microm, Walldorf, Germany), and the samples were cleaned in xylene. The sections were mounted using entellan. For negative control staining, the samples were processed in an identical manner, but incubation with the primary antibody was not done. The results of immunohistochemical staining were evaluated by a blinded observer under an Olympus BX40 (Tokyo, Japan) light microscope.

\section{TUNEL Assay}

Apoptotic cells were detected via the TUNEL assay. Nuclear deoxyribonucleic acid (DNA) fragmentation is an important morphological characteristic of apoptosis and can be detected in cells by TUNEL. An ApopTag Plus Peroxidase in situ apoptosis detection kit (S7101; Millipore, Billerica, MA, USA) was used according to the manufacturer's instructions. Briefly, after $20 \mu \mathrm{g} / \mathrm{mL}$ proteinase $\mathrm{K}$ treatment for 10 minutes at $37^{\circ} \mathrm{C}$ in a humidity chamber, the sections were treated with an equilibration buffer and were then incubated at $37^{\circ} \mathrm{C}$ with terminal deoxynucleotidyl transferase (TdT) for 1 hour. Following incubation with TdT, a stop wash buffer was applied to the sections for 10 minutes and the sections were washed in phosphate-buffered saline (PBS). Then, the sections were treated with an anti-digoxigenin peroxidase conjugate for 30 minutes and washed in PBS. After staining with 3, 3'-diaminobenzidine (DAB), counterstaining with Mayer's hematoxylin was performed. After mounting with entellan, TUNEL-positive cells were counted by a blinded observer under an Olympus BX40 light microscope.

\section{Statistical Analysis}

During the evaluation of the results, the immunostainings were identified with $\mathrm{H}$-scoring and determined as the ratio

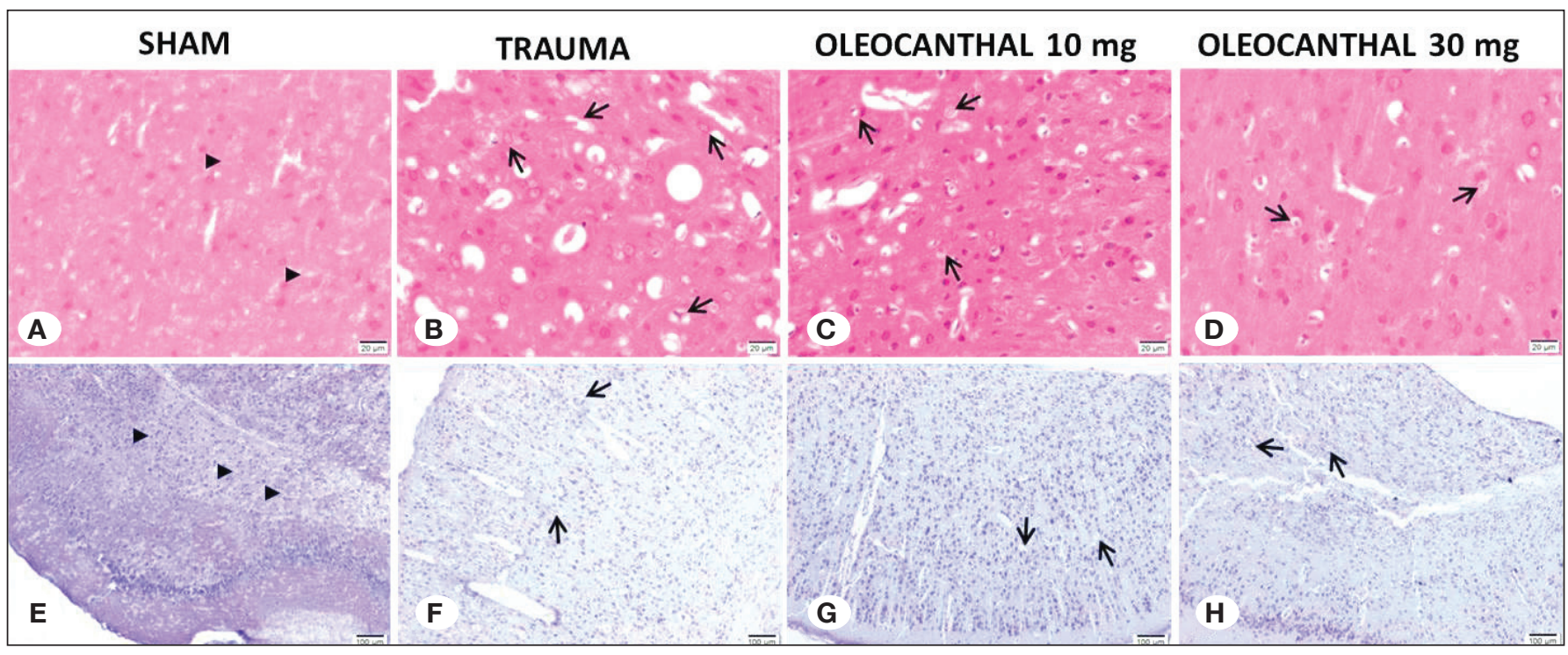

Figure 1: Hematoxylin \& Eosin (H\&E) and cresyl violet staining for each group. Shrunken cytoplasm, extensively dark pyknotic nuclei and vacuolization indicating tissue edema were demonstrated. In addition, occasional clumping of microglia and fusiform shaped microglia (arrows) are seen as degenerative changes in TBI compared to the control group (A and E). In the OC-treated samples (C, D, G, H), traumatic changes were less intense than in the trauma group (B and F). (A-D; H\&E, E-F; cresyl violet, scale bars $20 \mu \mathrm{m}$ in $\mathbf{A}$, B, C, D; scale bars $100 \mu \mathrm{m}$ in E, F, G, H). 
of positive-labeled cells to all cells in the chosen fields. Immunopositive cells were counted by a blinded observer as follows: 0 , no staining; 1 , weak staining; 2, moderate staining; 3 , strong staining in a fixed field. The respective score was then calculated using the following formula:

$\mathrm{H}-\mathrm{Score}=(\%$ stained cells at 0$) \times 0+(\%$ stained cells at $1+)$ $\times 1+(\%$ stained cells at $2+) \times 2+(\%$ stained cells at $3+) \times 3$.

The $\mathrm{H}$-score value varies from 0 to 300 (17).

For TUNEL staining, each section was counted for 100 TUNELpositive cells from randomly chosen fields. The apoptotic index was determined as the ratio of positive-labeled cells to all cells in the chosen fields. The percentage of apoptotic cells was calculated by a blinded observer as follows: 0 , no apoptosis; 1, 1\%-10\% apoptosis; 2, 11\%-25\% apoptosis; 3, 26\%-50\% apoptosis; 4, 51\%-75\% apoptosis; and 5, >75\% apoptosis $(15,16)$.

Biochemical and histological results were calculated on GraphPad (GraphPad Software, San Diego, CA, USA) using one-way analysis of variance and are presented as mean \pm standard deviation. Statistical significance was defined as $\mathrm{p} \leq 0.05$.

\section{- RESULTS}

\section{Histopathological Findings}

In the sham group, glial cells were diffusely localized in the brain cortex. The shape of the nucleus varied from round to rectangular. There were fewer oligodendrocytes than glial cells, and the tissue was perforated with various capillaries (Figure 1A). In the treated groups (Groups 2, 3 and 4), various degenerative changes were observed. Deterioration in cell shape such as dense nuclei and cytoplasm, enlargement of capillaries, increase in the number of myelinated axons, and vacuoles of different sizes were observed. These changes were seen in all treated groups, but less prominently in the $\mathrm{OC}$ treated groups (Figures 1B, C, D).

To better understand the mechanisms underlying this apparent neuroprotective effect, the cells were stained with eNOS, iNOS, VEGF, and S100 to determine oxidative stress, vascularization and gliosis (Figure 2). iNOS and eNOS are inflammatory products that are implicated in TBI.

For eNOS, which is an inflammatory product that is implicated in traumatic brain injury, statistically significant differences were seen between the sham group $(86 \pm 8.5)$ and the trauma group $(155 \pm 6.3)(p<0.001)$ and between the sham group and the groups treated with $10 \mathrm{mg} \mathrm{OC}(p<0.001)$ and $30 \mathrm{mg}$ OC $(p<0.001)$. In addition, there were significant differences between the trauma group and the groups treated with $10 \mathrm{mg}$ OC $(124 \pm 8.6)(p<0.001)$ and $30 \mathrm{mg} \mathrm{OC}(118 \pm 9)(\mathrm{p}<0.001)$. However, differences between the OC-treated groups were not significant ( $p>0.05)$.

For iNOS, which is an inflammatory product similar to eNOS, significant differences were observed between trauma group $(131 \pm 18)$ and the OC-treated groups $(p<0.001)$ and also between the trauma group and the group treated with $30 \mathrm{mg}$
OC $(115 \pm 12)(p<0.01)$, but not between the trauma group and the group treated with $10 \mathrm{mg}$ OC $(119 \pm 17)(p>0.05)$. The iNOS and eNOS staining intensity was reduced with OC treatment in a dose-dependent manner (Figure 3).

For VEGF, which demonstrated angiogenesis and endothelial cell proliferation, there were statistically significant differences between the sham group $(125 \pm 5)$ and the trauma group $(86 \pm 9)(p<0.001)$, between the trauma group and the group treated with $10 \mathrm{mg}$ OC $(100 \pm 8)(\mathrm{p}<0.05)$, and between the trauma group and the group treated with $30 \mathrm{mg} \mathrm{OC}(107 \pm 17)$ $(p<0.01)$. The VEGF staining intensity was the highest in the sham group and lowest in the trauma group. OC treatment increased the VEGF staining intensity in a dose-dependent manner (Figure 3).

For S100, which demonstrated gliosis, the comparison of OC-treated groups was not statistically significant ( $p>0.05$ ). However, the comparison of all other groups were statistically significant $(p<0.001)$ for the sham $(83 \pm 8)$ and trauma group $(159 \pm 11)$, the sham group and group treated with $10 \mathrm{mg} \mathrm{OC}$ $(126 \pm 12)$, and the trauma group vs. the group treated with 30 mg OC $(113 \pm 16): p<0.01$ for the sham group vs. the group treated with $30 \mathrm{mg} \mathrm{OC}$ and the trauma group vs. the group treated with $10 \mathrm{mg}$ OC. The $\mathrm{S} 100$ staining intensity was the highest in the trauma group. The staining intensity decreased with OC treatment in a dose-dependent manner (Figure 3).

Finally, TUNEL was used to evaluate apoptosis for explaining the relationship between oxidative stress and programmed cell death. Significant differences were seen between the sham group and the other groups $(p<0.001)$; for the sham group $(16 \% \pm 4.1)$ vs. the trauma group $(43 \% \pm 5)$, the sham group vs. the group treated with $10 \mathrm{mg} \mathrm{OC}(36 \% \pm 2.5)$, and the sham group vs. the group treated with $30 \mathrm{mg} \mathrm{OC}(26 \%$ \pm 4 ); $p<0.01$ for the trauma group vs. the group treated with $30 \mathrm{mg}$ OC (Figure 4). The TUNEL staining intensity was the highest in the trauma group and decreased with $\mathrm{OC}$ treatment in a dose-dependent manner. These results might show that $\mathrm{OC}$ treatment has a preventive effect against cell death.

\section{Biochemical Findings}

The average OSIs of blood samples were compared among all groups. In the treated groups, the OSI was the highest in the trauma group $(671 \pm 200)$ and was the lowest in the group treated with $30 \mathrm{mg} \mathrm{OC}(330 \pm 193)$. However, the difference was not statistically significant ( $p>0.05$ ). In the OC-treated groups, the average OSI decreased as the dose of OC increased, but the difference was not statistically significant (10 mg OC: 455 \pm 173 ) ( $p>0.05)$. The high OSI in the sham group (450 \pm 190$)$ (higher than that in the group treated with $30 \mathrm{mg} \mathrm{OC}$ ), in which only a skin incision was made, was thought to be a biological response to surgical skin incision. Table I and Figure 5 show the OSI values in rat's blood for each group.

\section{DISCUSSION}

In TBI, a robust inflammatory response is elicited. The posttraumatic period involves a cellular component and comprises the activation of resident glial cells, microglia, and astrocytes and the infiltration of blood leukocytes (26). These effects 
have an immediate impact on the brain by inducing necrotic cell loss as well as inducing apoptosis of the surrounding cells. Within minutes of these changes, a local inflammatory response occurs wherein astrocytes and microglia secrete pro-inflammatory cytokines, such as tumor necrosis factor, interleukin (IL)-6, and IL-1b, into the perilesional region. These pro-inflammatory cytokines mobilize immune and glial cells to the injury site, causing edema and further inflammation. This phase is associated with gliosis, demyelination, and continued apoptosis (6). To date, many agents have been used to prevent secondary injury after TBI. Although several investigations have shown the effectiveness of some agents in preclinical studies, to our knowledge, their effectiveness in clinical trials has not been observed. Still, no pharmacological treatment has been found to confer neuroprotection by targeting secondary injury mechanisms (20). In this study, we used $\mathrm{OC}$ in rats to evaluate whether it has neuroprotective effects after TBI or not.

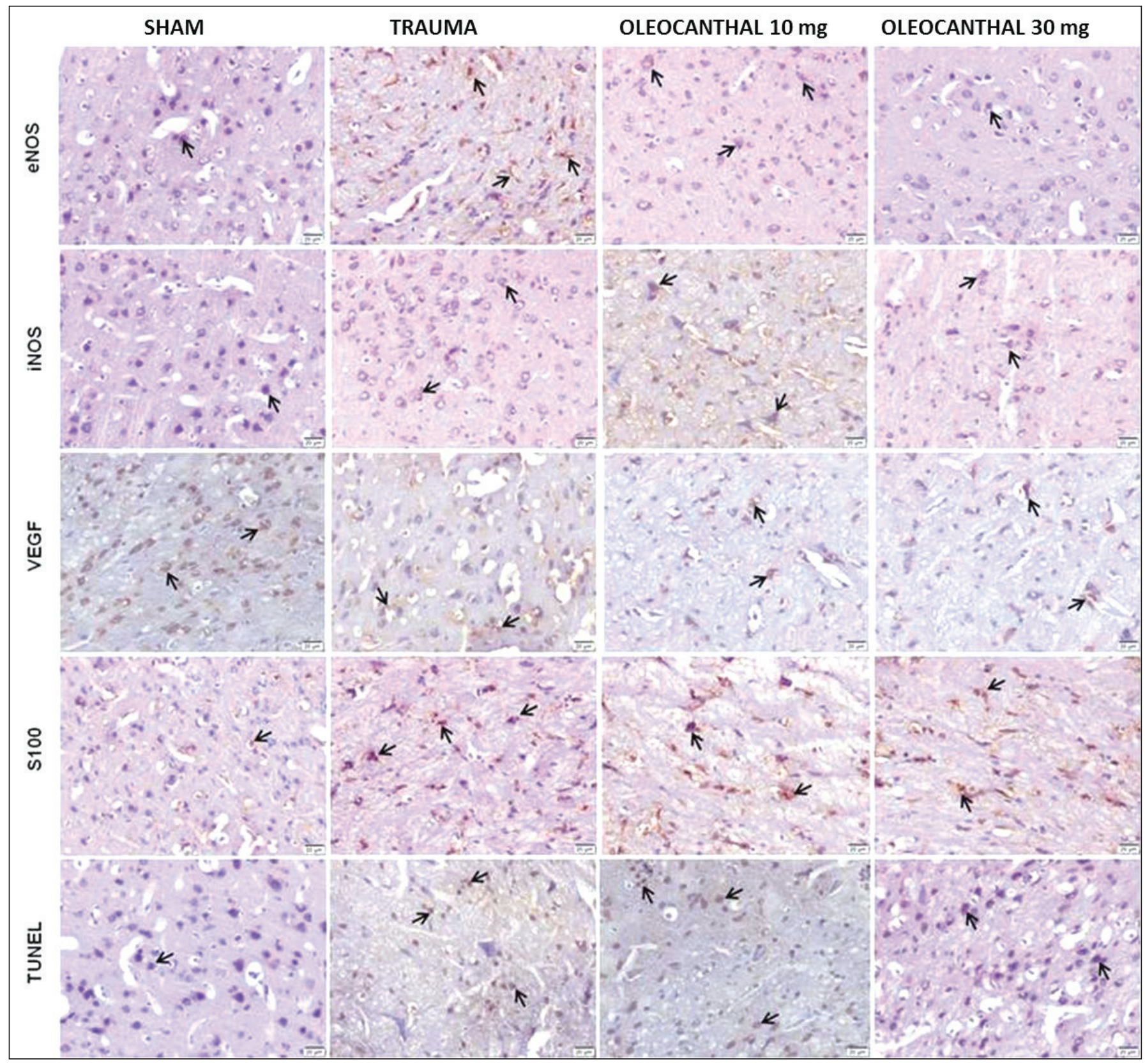

Figure 2: Immunocytochemical staining of anti-eNOS, anti-iNOS, anti-VEGF, S100 are seen. TUNEL stainings for each group is seen at the bottom of the figure. The NOS immunopositivity was increased in neurons after TIB. Apoptotic cells with pyknotic nuclei and apoptotic bodies were detected. TUNEL, NOS and S100 staining of TBI were increased compared those of sham and they were decreased with OC treatment compared to those of trauma. VEGF staining were decreased after TBI and increased after OC treatment (scale bars $20 \mu \mathrm{m}$ ). 

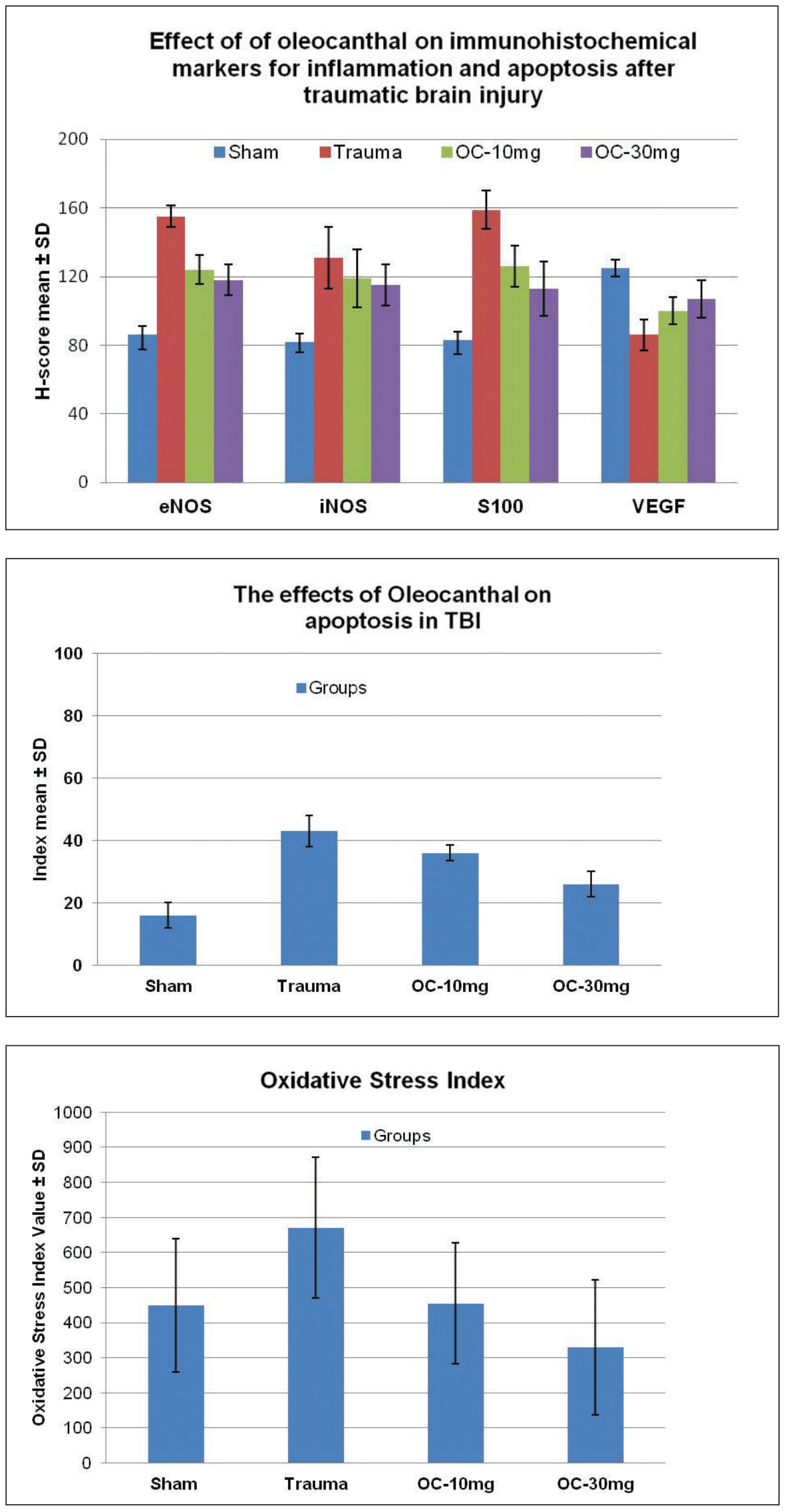

Figure 3: Effect of OC on immunohistochemical markers and apoptosis for inflammation after traumatic brain injury. eNOS, iNOS, and S100 levels decreased with OC treatment in a dosedependent manner. VEGF levels increased with $\mathrm{OC}$ treatment in a dose-dependent manner.
Figure 4: Effect of OC on apoptosis after TBI. The amount of apoptosis that is noticed in the trauma group shows a decrease in the OC-treated groups in a dose-dependent manner.
Figure 5: Graphic demonstrated the average OSI for each group. The OSI decreased with OC treatment in a dose-dependent manner. The high OSI in the sham group (higher than in the group treated with $30 \mathrm{mg} \mathrm{OC)}$, in which only a skin incision was made, was thought to be a biological response to the surgical skin incision. 
OC is one of the phenolic compounds (approximately 10\%) in VOO, which has recently emerged as a potential therapeutic molecule for different conditions, including inflammation, cancers, and neurodegenerative diseases (21). VOO is found in the Mediterranean diet and daily mediterranean diet which typically includes an intake of $25-50 \mathrm{~mL}$ VOO (5). OC was first identified by Andrewes et al. in 2003 (2). It shares the same mechanistic anti-inflammatory action with ibuprofen. Therefore, it is currently acknowledged as a naturally occurring NSAID $(2,19)$. Beauchamp et al. (3), and others have reported that $\mathrm{OC}$ inhibits cyclooxygenases 1 and 2 in a dosedependent manner and that it is more effective than ibuprofen in inhibiting these enzymes at equimolar concentrations $(18,21)$. In addition, Scotece et al. demonstrated that $O C$ inhibits other pro-inflammatory factors such as macrophage inflammatory protein-1a and IL-6 in J774 macrophages and ATDC5 chondrocytes (22). Furthermore, OC attenuates inflammatory mediators, such as iNOS, which play a role in the pathogenesis of degenerative joint disease (11). Secondary inflammation involves oxidative stress, and the upregulation of the inflammatory enzyme iNOS leads to the formation of free radicals, such as reactive oxygen species (10), which can be activated by apoptosis. In the present study, we showed that the production of eNOS and iNOS were significantly decreased with $\mathrm{OC}$ treatment in a dose-dependent manner. Further, the TUNEL staining (TUNEL staining was initially described as a method for staining cells that have undergone programmed cell death, or apoptosis) intensity was the highest in the trauma group and significantly decreased with OC treatment in a dose-dependent manner. These results could be interpreted in the following manner: OC inhibits the formation of reactive oxygen species by reducing oxidative stress and preventing cell death.

In many studies, S100 and VEGF are preferred markers to determine trauma severity $(13,23,24)$. $S 100$ has been considered a glial marker protein and it has also been detected in other cell types such as ependymal cells and the choroid plexus epithelium. S100 marker has been investigated immunohistochemically in autopsy cases with regard to the cause of death, the severity of TBI and the survival times (13). VEGF is a trophic factor expressed in the central nervous system after injury, and VEGF expression inhibition after injury may actually exacerbate outcomes $(23,24)$. Therefore, we used S100 and VEGF markers in addition to eNOS, iNOS, and TUNEL staining to determine OC's protective effect against $\mathrm{TBI}$. The production of $\mathrm{S} 100$ was highest in the trauma group and significantly decreased with $\mathrm{OC}$ treatment in a dose-dependent manner. This indicates that OC treatment reduced gliosis and had a neuroprotective effect after TBI. In addition, the VEGF staining intensity was the lowest in the trauma group and significantly increased with OC treatment in a dose-dependent manner. This could be interpreted in the following manner: OC treatment accelerates the recovery of brain damage by stimulating the formation of new vessels.

\section{CONCLUSION}

Our results indicated that OC has a protective effect on neural cells after TBI. This effect is achieved by reducing oxidative stress and apoptosis. OC reduced the plasma oxidative stress status in a dose-dependent manner. To our knowledge, this is the first study to evaluate the neuroprotective effects of $\mathrm{OC}$ in a rat model of TBI. However, further experimental and clinical studies will be needed to understand its effects on neurons after TBI.

\section{ACKNOWLEDGEMENT}

Preparation for publication of this article was partly supported by the Turkish Neurosurgical Society.

\section{REFERENCES}

1. Abuznait AH, Qosa H, Busnena BA, El Sayed KA, Kaddoumi A: Olive-oil-derived oleocanthal enhances $\beta$-amyloid clearance as a potential neuroprotective mechanism against Alzheimer's disease: In vitro and in vivo studies. ACS Chem Neurosci 4(6): 973-982, 2013

2. Andrewes $P$, Busch JL, de Joode $T$, Groenewegen $A$, Alexandre $\mathrm{H}$ : Sensory properties of virgin olive oil polyphenols: Identification of deacetoxy-ligstroside aglycon as a key contributor to pungency. J Agric Food Chem 51(5):14151420, 2003

3. Beauchamp GK, Keast RS, Morel D, Lin J, Pika J, Han Q, Lee $\mathrm{CH}$, Smith AB, Breslin PA: Phytochemistry: Ibuprofen-like activity in extra-virgin olive oil. Nature 437(7055):45,46, 2005

4. Centers for Disease Control and Prevention National Center for Injury Prevention and Control. Traumatic brain injury in the United States: A report to Congress. Available at: https:// www.cdc.gov/traumaticbraininjury/pdf/tbi_in_the_us.pdf. Accessed January 28, 2017

5. Corona G, Spencer JP, Dessì MA: Extra virgin olive oil phenolics: Absorption, metabolism, and biological activities in the Gl tract. Toxicol Ind Health 25(4-5):285-293, 2009

6. Dixon KJ: Pathophysiology of traumatic brain injury. Phys Med Rehabil Clin N Am 28(2):215-225, 2017

7. Erel O: A new automated colorimetric method for measuring total oxidant status. Clin Biochem 38:1103-1111, 2005

8. Erel O: A novel automated direct measurement method for total antioxidant capacity using a new generation, more stable ABTS radical cation. Clin Biochem 37:277-285, 2004

9. Ersoy B, Ozbilgin K, Kasirga E, Inan S, Coskun S, Tuglu I: Effect of growth hormone on small intestinal homeostasis relation to cellular mediators IGF-I and IGFBP-3. World J Gastroenterol 15(43):5418-5424, 2009

10. Günther M, Davidsson J, Plantman S, Norgren S, Mathiesen T, Risling M: Neuroprotective effects of $\mathrm{N}$-acetylcysteine amide on experimental focal penetrating brain injury in rats. J Clin Neurosci 22(9): 1477-1483, 2015

11. lacono A, Gómez R, Sperry J, Conde J, Bianco G, Meli R, Gómez-Reino JJ, Smith AB 3rd, Gualillo O: Effect of oleocanthal and its derivatives on inflammatory response induced by lipopolysaccharide in a murine chondrocyte cell line. Arthritis Rheum 62(6):1675-1682, 2010

12. Kosecik M, Erel O, Sevinc E, Selek S: Increased oxidative stress in children exposed to passive smoking. Int J Cardiol 100:61-64, 2005 
13. Krohn M, Dreßler J, Bauer M, Schober K, Franke H, Ondruschka B: Immunohistochemical investigation of S100 and NSE in cases of traumatic brain injury and its application for survival time determination. J Neurotrauma 32(7):430-440, 2015

14. McGinn MJ, Povlishock JT: Pathophysiology of traumatic brain injury. Neurosurg Clin N Am 27(4):397-340, 2016

15. Mete M, Aydemir I, Tuglu IM, Selcuki M: Neurotoxic effects of local anesthetics on the mouse neuroblastoma NB2a cell line. Biotech Histochem 90(3):216-22, 2015

16. Mete M, Aydemir I, Unsal UU, Duransoy YK, Tuglu IM, Selcuki M: Neuroprotective effects of bone marrow-derived mesenchymal stem cells and conditioned medium in mechanically injured neuroblastoma cells. Turk J Med Sci 46(6):1900-1907, 2016

17. Numata M, Morinaga S, Watanabe T, Tamagawa $H$, Yamamoto N, Shiozawa M, Nakamura Y, Kameda Y, Okawa S, Rino Y, Akaike M, Masuda M, Miyagi Y: The clinical significance of SWI/SNF complex in pancreatic cancer. Int J Oncol 42(2): 403-410, 2013

18. Parkinson L, Cicerale S: The health benefiting mechanisms of virgin olive oil phenolic compounds. Molecules 21(12): E1734, 2016

19. Parkinson L, Keast R: Oleocanthal, a phenolic derived from virgin olive oil: A review of the beneficial effects on inflammatory disease. Int J Mol Sci 15(7):12323-12334, 2014
20. Pearn ML, Niesman IR, Egawa J, Sawada A, Almenar-Queralt A, Shah SB, Duckworth JL, Head BP: Pathophysiology associated with traumatic brain injury: Current treatments and potential novel therapeutics. Cell Mol Neurobiol 37(4):571585,2017

21. Scotece M, Conde J, Abella V, Lopez V, Pino J, Lago F, Smith AB 3rd, Gómez-Reino JJ, Gualillo O: New drugs from ancient natural foods. Oleocanthal, the natural occurring spicy compound of olive oil: A brief history. Drug Discov Today 20(4):406-410, 2015

22. Scotece M, Gómez R, Conde J, Lopez V, Gómez-Reino JJ, Lago F, Smith AB $3^{\text {rd }}$, Gualillo O: Further evidence for the antiinflammatory activity of oleocanthal: Inhibition of MIP-1a and IL-6 in J774 macrophages and in ATDC5 chondrocytes. Life Sci 91(23-24): 1229-1235, 2012

23. Sköld MK, Risling M, Holmin S: Inhibition of vascular endothelial growth factor receptor 2 activity in experimental brain contusions aggravates injury outcome and leads to early increased neuronal and glial degeneration. Eur J Neurosci 23(1):21-34, 2006

24. Thau-Zuchman O, Shohami E, Alexandrovich AG, Leker RR: Vascular endothelial growth factor increases neurogenesis after traumatic brain injury. J Cereb Blood Flow Metab 30(5): 1008-1016, 2010

25. Ucar T, Tanriover G, Gurer I, Onal MZ, Kazan S: Modified experimental mild traumatic brain injury model. J Trauma 60(3):558-565, 2006

26. Woodcock T, Morganti-Kossmann MC: The role of markers of inflammation in traumatic brain injury. Front Neurol 4:18, 2013 